\title{
On the stability of Saturn's rings to gravity disturbances
}

\author{
E. Griv ${ }^{1}$, M. Gedalin ${ }^{1}$, and C. Yuan ${ }^{2}$ \\ 1 Department of Physics, Ben-Gurion University of the Negev, PO Box 653, Beer-Sheva 84105, Israel \\ 2 Academia Sinica Institute of Astronomy and Astrophysics (ASIAA), PO Box 23-141, Taipei, Taiwan
}

Received 7 August 2002 / Accepted 20 December 2002

\begin{abstract}
A method for investigating the small-amplitude nonlinear oscillations of low and moderately high optical depth regions of Saturn's main rings is developed through the using of the Boltzmann kinetic equation with a Krook model integral of interparticle collisions and the Poisson equation. A mathematical formalism in the approximation of weak turbulence (a quasilinearization of the Boltzmann equation) is developed. Conditions under which the quasilinear approximation can be used to describe wave-particle interactions are calculated with reference to the excitation of Jeans-type gravity disturbances (those produced by a spontaneous perturbation and/or a companion system). It is shown that the spontaneous, almost aperiodically growing Jeans-unstable spiral gravity oscillations developing in the disk's plane must influence the distribution of mutually gravitating particles in such a way as to hinder the oscillations excitation, i.e., to increase the spread of random velocities. As a result, finally in the disk there can be established a quasi-stationary distribution so that the Jeans-unstable density waves are completely vanishing. Thus, in the nonlinear regime, the particles can continue developing gravity-unstable density condensations only if some effective mechanism of "cooling" exists. We suggest that in Saturn's rings the cooling mechanism leading to the long-term density waves activity is actually operating: inelastic (dissipative) collisions reduce the magnitude of the relative velocity of particles.
\end{abstract}

Key words. planets and satellites: individual: Saturn - planets: rings

\section{Introduction}

The rapidly and differentially rotating Saturnian ring disk of mutually gravitating particles is highly dynamic and is subject to various collective instabilities of gravity disturbances (Goldreich \& Tremaine 1982; Shu 1984; Griv et al. 2000). This is because the evolution of the system is primarily driven by angular momentum redistribution (Goldreich \& Tremaine 1982). A gravitational Jeans-type instability (and indeed also embedded and external Saturn's moons, charged particles, and micrometeoroid impacts; Cuzzi et al. 1981; Lissauer 1981; Shu et al. 1983; Goertz \& Morfill 1988; Durisen 1995; Lewis \& Stewart 2000; Spahn \& Sremcevic 2000) plays a fundamental role in sculpting of elaborate ring system of Saturn. Authors who have investigated the Jeans instability in Saturn's rings have usually been interested in the linear theory. The investigations carried out in the linear approximation, however, allow us to determine only the spectrum of the excited oscillations and their growth rates during the first stages of the excitation. But the theory remains incomplete. Other problems, which can be treated only within the framework of nonlinear theory, are account of the reaction of the oscillations on the equilibrium parameters of the system and the determination of the amplitude of the oscillations that are produced.

Send offprint requests to: E. Griv, e-mail: griv@bgumail.bgu.ac.il
In this paper, for the first time as far as we are aware, the quantitative weakly nonlinear (quasilinear) theory of Jeans oscillations of Saturn's rings is developed. The method that we adopt follows the development of Griv et al. (2000, 2001, 2002). Conditions for the applicability of quasilinear theory of Jeans instability in Saturn's rings are given in Appendix. Generally speaking, the quasilinear approach, i.e., the case when the dynamics of the system can be described in the language of weakly interacting linear waves, can be justified if the energy in the excited spectrum is small compared with the total mechanical energy in particles but large compared to thermal noise ${ }^{1}$. Accordingly, there are many random collective oscillations present in the system and it is permissible to treat the phases of these oscillations as being random in some sense. Arbitrary disturbances can be expressed as a superposition of eigenmodes, with each eigenmode evolving independently. The theory of strong turbulence is still far from complete. As shown in the present paper, the quasilinear theory can be applied to aperiodic Jeans-type instabilities we are interested in, provided that in the initial state the system parameters deviate little from the critical values at which instability sets in.

\footnotetext{
1 An original version of quasilinear theory was proposed in the pioneering works by Vedenov et al. (1962) and Drummond \& Pines (1962). See Lifshitz \& Pitaevskii (1981, p. 199), Galeev \& Sagdeev (1983), and Swanson (1989, p. 291) as reviews.
} 
We use the method of gas-kinetic theory which incorporates interparticle collisions through a Krook phenomenological integral of collisions (Shu \& Stewart 1985; Griv \& Chiueh 1997; Griv et al. 1997). In particular, we investigate in detail the important limit of rare collisions between particles. The results of the analysis are applied to Saturn's rings: we predict the irregular, recurrent $\sim 100 \mathrm{~m}$ or so structure in low and moderately high optical depth regions ( $\tau \leq 1$, where $\tau$ is the normal optical depth) of Saturn's main A, B, and C rings.

Salo (1992, 1995), Richardson (1994), Osterbart \& Willerding (1995), Sterzik et al. (1995), Griv (1998), Daisaka \& Ida (1999), and Ohtsuki \& Emori (2000) already predicted such a fine-scale structure in Saturn's rings by $N$-body simulations. Our significant contribution is just a kinetic theory derivation of results obtained before in $N$-body simulations or through other analytic approximations. Earlier, Colombo et al. (1976) and Franklin \& Colombo (1978) have explained the so-called quadrupole asymmetry observed in the bright portions of ring A (e.g., Franklin et al. 1987 and Dones et al. 1993) in terms of numerous unresolved Julian-Toomre (Julian \& Toomre 1976) spiral density wakes excited around massive ring particles. These forced waves studied by Colombo et al. and Franklin \& Colombo are not to be confused, of course, with Lin \& Shu's (Lin \& Shu 1966; Lin et al. 1969; Shu 1970; Binney \& Tremaine 1987, p. 360) fully self-consistent density wave proposal explored in our theory. In a way of contrast, our model relies on normal modes of collective oscillations of a particulate disk to maintain spiral coherence at all radii. These modes are associated with such phenomena as, for example, the appearance of the spiral structure of galaxies (Lin \& Shu 1966; Lin et al. 1969; Shu 1970; Binney \& Tremaine 1987, p. 336; Bertin \& Lin 1996; Griv \& Peter 1996; Griv et al. 1999, 2001, 2002).

\section{Basic equations}

In the spirit of Griv et al. (2000), we solve a self-consistent system of the Boltzmann kinetic equation and the Poisson equation describing the motion of a self-gravitating ensemble of randomly colliding particles, looking for waves which propagate in a two-dimensional rotating disk. The approximation of an infinitesimally thin disk is a valid approximation if one considers disturbances with a radial wavelength that is greater $h$, the typical disk thickness (Safronov 1960, 1980; Toomre 1964; Goldreich \& Lynden-Bell 1965; Lin \& Shu 1966; Lin et al. 1969; Shu 1970, 1984; Vandervoort 1970; Morozov 1981a). In Saturn's main rings, $h=5-50 \mathrm{~m}$ (Esposito 1986, 1993; Poulet et al. 2000). It has also been shown by $N$-body simulations that the inclusion of motions normal to the plane makes little difference to the evolution of the rotating thin disk (Hohl 1978; Griv \& Chiueh 1998). This justifies the two-dimensional treatment of the main part of a rapidly rotating disk ${ }^{2}$.

\footnotetext{
${ }^{2}$ Self-gravitating evolution of a thick disk is generally very similar to that of a razor-thin disk, because the induced motions are almost planar. One qualitative difference the disk's finite but small thickness makes is that it tends to be stabilizing by reducing self-gravity at the midplane (Vandervoort 1970; Morozov 1981a; Shu 1984).
}

We assume that the particles move in the disk plane so that $v_{z}=0$. This allows us to use in a circular rotating frame the two-dimensional distribution function $f\left(r, \varphi, v_{r}, v_{\varphi}, t\right)$ such that $\tilde{f}=f\left(r, \varphi, v_{r}, v_{\varphi}, t\right) \delta(z) \delta\left(v_{z}\right), f=\int \tilde{f} \mathrm{~d} z \mathrm{~d} v_{z}$, and $\int f \mathrm{~d} v_{r} \mathrm{~d} v_{\varphi}=\sigma$, where $\sigma(\boldsymbol{r}, t)$ is the surface density, $v_{r}, v_{\varphi}, v_{z}$ are the small residual (random) velocities, $r, \varphi, z$ are the planetocentric cylindrical coordinates and the axis of disk rotation is taken oriented along the $z$-axis, and $t$ is time.

The local distribution function of identical particles $f(\boldsymbol{r}, \boldsymbol{v}, t)$ must satisfy the Boltzmann equation (with a Krook integral of collisions)

$\frac{\partial f}{\partial t}+\boldsymbol{v} \cdot \frac{\partial f}{\partial \boldsymbol{r}}-\nabla \Phi \cdot \frac{\partial f}{\partial \boldsymbol{v}}=-v_{\mathrm{c}}\left(f-f_{0}\right)$.

In Eq. (1), the total azimuthal velocity of the particles may be represented as a sum of $v_{\varphi}$ and the basic circular velocity $r \Omega$, and $\left|v_{r}\right|,\left|v_{\varphi}\right| \ll r \Omega$. Here and below, the quantities $\Omega(r)$ and $\kappa(r)=2 \Omega[1+(r / 2 \Omega)(\mathrm{d} \Omega / \mathrm{d} r)]^{1 / 2}$ denote the angular velocity of disk rotation and the epicyclic frequency at the distance $r$ from the Saturn, and $\kappa=\Omega$ for a Keplerian rotation. Also, $\Phi(r, t)$ is the total gravitational potential determined selfconsistently from the Poisson equation

$\Delta \Phi=4 \pi G \int f \mathrm{~d} v=4 \pi G \sigma \delta(z)$,

where $\delta(z)$ is the Dirac delta-function with respect to the spatial coordinate $z$. In Eq. (1), $f$ is the actual distribution function, $f_{0}$ is the steady-state (Maxwellian) equilibrium distribution function, $v_{\mathrm{c}}=n\langle s v\rangle$ plays the role of the velocity-independent collision frequency, $n$ is the number density of particles, $s$ is the effective radius of a particle, and $\langle\cdots\rangle$ denotes the average over particles of all random velocities $v$ in a Maxwellian distribution. The Krook integral $-v_{\mathrm{c}}\left(f-f_{0}\right)$ is called a model because it cannot be derived from the exact Boltzmann integral but can only be constructed by general physical reasoning, i.e., the need to satisfy the laws of conservation of particle number, momentum, and energy (cf. the so-called tau-approximation widely used in physical kinetics; Lifshitz \& Pitaevskii 1981).

Some simple relations exist between optical depth, ring thickness, particle density, and other quantities that define the physical state of a planetary ring. Thus the optical depth $\tau$ is approximately equal to the ratio of the collision frequency $v_{\mathrm{c}}$ to the orbital frequency $\Omega$ :

$\tau \approx v_{\mathrm{c}} / \Omega$

(Jeffreys 1947; Cook \& Franklin 1964; Goldreich \& Tremaine 1982; Bridges et al. 1984; Shu 1984; Stewart et al. 1984; Araki 1991). This is the fundamental result which was nicely rediscovered by Shu \& Stewart (1985), employing a Krook approximation to the collision integral.

We assume that (i) there are only binary physical collisions, and momentum is conserved in collisions; and (ii) there is no correlation in motion between the colliding species, that is, Boltzmann's hypothesis of molecular chaos is adopted (cf. the assumptions undelying the Goldreich \& Tremaine 1978 standard model). A case of rare $\left(\Omega \approx \kappa>v_{\mathrm{c}}\right)$ interparticle collisions is considered. Then the collisions cause only small corrections of the perturbed distribution function and in the 
zero-order approximation of the theory all dissipative effects may be ignored. Such low and moderately high optical depth regions with relatively rare collisions between particles can be found in the main part of Saturn's rings: in the inner C ring at distances $r<92000 \mathrm{~km}$ from Saturn's center, in the inner portions of the densest B ring at distances $r<100000 \mathrm{~km}$, and in the outer A ring at distances $r>122000 \mathrm{~km}$ (Esposito 1986, 1993; Nicholson et al. 2000). The density and the collision frequency of the central B ring at distances $100000 \mathrm{~km}<r<$ $122000 \mathrm{~km}$ from Saturn's center probably suggest that it be modeled not as a rarefied gas, as in the Goldreich \& Tremaine (1978) model, but as a condensed gas (Araki 1991).

We apply the standard procedure of the quasilinear approach (Sagdeev \& Galeev 1969; Galeev \& Sagdeev 1983; Alexandrov et al. 1984; Krall \& Trivelpiece 1986; Swanson 1989). In the quasilinear theory, one may follow the ordinary procedure of linearization of Eq. (1) by writing $f=f_{0}(\boldsymbol{v}, t)+$ $f_{1}(\boldsymbol{r}, \boldsymbol{v}, t)$ and $\Phi=\Phi_{0}(r, t)+\Phi_{1}(\boldsymbol{r}, t)$, where $\left|f_{1} / f_{0}\right| \ll 1$ and $\left|\Phi_{1} / \Phi_{0}\right| \ll 1$. The functions $f_{1}$ and $\Phi_{1}$ are functions oscillating rapidly in space and time, while the functions $f_{0}$ and $\Phi_{0}$ describe the slowly (and monotonically) developing spatially homogeneous "background" against which small disturbances develop. According to the definition the distribution function $f_{0}$ must be nonnegative. The distribution $f_{0}$ continues to distort as long as the distribution is unstable; $f_{0}(t=0) \equiv f_{\mathrm{e}}$ and $\Phi_{0}(t=0) \equiv \Phi_{\mathrm{e}}$, where $f_{\mathrm{e}}$ and $\Phi_{\mathrm{e}}$ are the equilibrium distribution function and the gravitational potential. Thus, we suppose that the nonlinear effects in Saturn's are small, so that the linear theory is a good first approximation.

Linearizing Eq. (1) and separating fast and slow varying variables one obtains the equation for the small fast developing part of the distribution function $f_{1}(\boldsymbol{r}, \boldsymbol{v}, t)$

$\frac{\mathrm{d} f_{1}}{\mathrm{~d} t}=\frac{\partial \Phi_{1}}{\partial r} \frac{\partial f_{0}}{\partial v_{r}}+\frac{1}{r} \frac{\partial \Phi_{1}}{\partial \varphi} \frac{\partial f_{0}}{\partial v_{\varphi}}-v_{\mathrm{c}} f_{1}$,

where $\mathrm{d} / \mathrm{d} t$ means total derivative along the particle orbit. The equation for the large slowly developing part of the distribution function $f_{0}(\boldsymbol{v}, t)$ is

$\frac{\partial f_{0}}{\partial t}=\left\langle\frac{\partial \Phi_{1}}{\partial r} \frac{\partial f_{1}}{\partial v_{r}}+\frac{1}{r} \frac{\partial \Phi_{1}}{\partial \varphi} \frac{\partial f_{1}}{\partial v_{\varphi}}\right\rangle$,

where $\langle\cdots\rangle$ denotes a time average over the fast oscillations, $\langle f\rangle=f_{0},\langle\Phi\rangle=\Phi_{0}$, and $\left\langle f_{1}\right\rangle=\left\langle\Phi_{1}\right\rangle=0$. Of course, the inequality $f_{0}(\boldsymbol{v}, t) \gg\left|f_{1}(\boldsymbol{r}, \boldsymbol{v}, t)\right|$ was needed and small nonlinear terms were neglected in the derivation of Eqs. (3) and (4).

\section{Oscillation spectra}

To determine oscillation spectra, let us consider the stability problem in the local WKB approximation (e.g., Krall \& Trivelpiece 1986, p. 424); this is accurate for short wave disturbances only, but qualitatively correct even for disturbances with a longer wavelength, of the order of the disk radius $R$. In the local WKB approach the azimuthally-averaged properties of the system are taken to vary with position on a lengthscale much larger than that of the spiral/radial features. This condition is very well met in Saturn's rings. In the local WKB approximation, assuming the weakly inhomogeneous disk, the disturbance of equilibrium parameters is selected in the form of a superposition of different harmonics corresponding to different modes of disk oscillations (in the circular rotating frame)

$\boldsymbol{\aleph}_{1}(\boldsymbol{r}, t)=\sum_{\boldsymbol{k}} \boldsymbol{\aleph}_{k} \mathrm{e}^{i k_{k} r+i m \varphi-i \omega_{*, k} t}+$ c.c.,

where $\boldsymbol{\aleph}_{k}$ is an amplitude that is a constant in space and time, $k_{r}$ is the constant radial wavenumber, $m$ is the nonnegative azimuthal mode number (=number of spiral arms), $\varphi$ denotes longitude increasing in the direction of disk rotation, $\omega_{*}=\omega-m \Omega$ is the Doppler-shifted complex wavefrequency, $\omega_{*, \boldsymbol{k}}=\mathfrak{R} \omega_{*, \boldsymbol{k}}+i \mathfrak{J} \omega_{*, \boldsymbol{k}}$, suffixes $\boldsymbol{k}$ denote the $\boldsymbol{k}$ th Fourier component, $\left|k_{r}\right| R \gg 1, R=140000 \mathrm{~km}$ is the typical radius of the system, and c.c. means the complex conjugate. In the linear theory, one can select one of the Fourier harmonics: $\aleph_{k} \mathrm{e}^{i k_{r} r+i m \varphi-i \omega_{*} t}$. The existence of solutions with $\mathfrak{J} \omega_{*}$ greater than zero implies instability of small-amplitude gravity disturbances (those produced by a spontaneous disturbance and/or a companion system). For a given mode, the (almost) constant phase velocity of density waves is $\Omega_{\mathrm{p}}=\mathfrak{R} \omega_{*} / m$ called the pattern speed. Since $\mathfrak{R} \omega_{*}$ is a constant, independent of time or radius, each component will remain identical with time. Thus, the imaginary part of the frequency of excited waves $\omega_{*}$ corresponds to a growth or decay of the components in time and the real part to a rotation with constant angular velocity $\Omega_{\mathrm{p}}$.

To integrate Eq. (3) over $t$, one needs the expressions for unperturbed particle orbits in the equilibrium central field. Such unperturbed epicyclic trajectories are given by (Griv 1996; Griv \& Peter 1996; Grivnev 1988)

$$
\begin{aligned}
& r=r_{0}+\frac{v_{\perp}}{\kappa}\left[\sin \phi_{0}-\sin \left(\phi_{0}-\kappa t\right)\right], \quad v_{r}=\frac{\mathrm{d} r}{\mathrm{~d} t}, \\
& \varphi=\Omega t-\frac{2 \Omega}{\kappa} \frac{v_{\perp}}{r \kappa}\left[\cos \phi_{0}-\cos \left(\phi_{0}-\kappa t\right)\right], \\
& v_{\varphi}=r \frac{\mathrm{d} \varphi}{\mathrm{d} t}-r \Omega \approx r \frac{\mathrm{d} \varphi}{\mathrm{d} t}-r \Omega-r\left(r-r_{0}\right) \frac{\mathrm{d} \Omega}{\mathrm{d} r}
\end{aligned}
$$

where $v_{\perp}, \phi_{0}$ are constants of integration, $v_{\perp} / \kappa r_{0} \approx \rho / r_{0} \ll 1$, $\rho \sim v_{\perp} / \kappa$ is the mean epicyclic radius, the $r$-derivatives are evaluated at $r=r_{0}$, and $v_{r}, v_{\varphi}$ are the components of the particle's velocity relative to the local (rotating) standard of rest. The orbits (6) and (7) separate regular circular and random motion components, and contain a directional motion of particles (basic circular rotation proportional to $\Omega$ ) and oscillations with the epicyclic frequency $\kappa$. The angular velocity $\Omega$ and epicyclic frequency $\kappa$ are evaluated at $r_{0}$. The motion along the epicycle is proceeds in the opposite direction to the primary rotation (to conserve an angular momentum). Of course, only in the limit $\kappa>v_{\mathrm{c}}$ can one speak of the epicyclic rotation.

Paralleling the analysis leading to Eq. (12) in Griv et al. (2000), from Eqs. (3) and (5) it is straightforward to show that the perturbed distribution function is

$f_{1}=\frac{\Phi_{k}}{v_{\perp}} \frac{\partial f_{0}}{\partial v_{\perp}} \sum_{l=-\infty}^{\infty} \mathrm{e}^{i\left(l\left(k-\omega_{*}-i v_{\mathrm{c}}\right) t\right.} \frac{l \kappa J_{l}^{2}(\chi)}{l \kappa-\omega_{*}-i v_{\mathrm{c}}}+$ c.c.

where $v_{\perp}^{2}=v_{r}^{2}+(2 \Omega / \kappa)^{2} v_{\varphi}^{2}, J_{l}(\chi)$ is the Bessel function of the first kind with its argument $\chi=k_{*} v_{\perp} / \kappa$,

$k_{*}=k\left\{1+\left[(2 \Omega / \kappa)^{2}-1\right] \sin ^{2} \psi\right\}^{1 / 2}$ 
is the effective wavenumber, $\psi=\arctan \left(m / k_{r} r\right)$ is the pitch angle of disturbances, $k=\sqrt{k_{r}^{2}+(m / r)^{2}}$ is the total wavenumber, both $\kappa$ and $\left|\omega_{*}\right| \gg v_{\mathrm{c}}$, and $f_{0} \equiv f_{\mathrm{e}}$ is a given basic distribution function determined from the equation $\partial f_{0}(t=0) / \partial t=0$. In Eq. (8) the denominator vanishes when $\mathfrak{R} \omega_{*}-l_{\kappa}=0$. This occurs near corotation $\left(l=0, \mathfrak{R} \omega_{*}=0\right)$ and other resonances.

Integrating Eq. (8) over velocity space and equating the result to the perturbed surface density given by the solution of the Poisson Eq. (2)

$\sigma_{1}=-|k| \Phi_{1} / 2 \pi G+$ c.c.

the generalized Lin-Shu type dispersion relation (Lin \& Shu 1966; Lin et al. 1969; Shu 1970; Binney \& Tremaine 1987, p. 352) in the plane $z=0$ may be obtained (Griv et al. 2000; see also Morozov 1980, 1981b; Griv \& Peter 1996; Griv et al. 1999)

$$
\frac{k^{2} c_{r}^{2}}{2 \pi G \sigma_{0}|k|}=-\kappa \sum_{l=-\infty}^{\infty} l \frac{\mathrm{e}^{-x} I_{l}(x)}{\omega_{*}+i v_{\mathrm{c}}-l \kappa},
$$

where $\omega_{*} \neq l \kappa, \sigma_{0}$ is the equilibrium surface mass density, $x=k_{*}^{2} c_{\mathrm{r}}^{2} / \kappa^{2} \approx k_{*}^{2} \rho^{2}, c_{\mathrm{r}}$ is the radial dispersion of random velocities, $\rho=c_{\mathrm{r}} / \kappa$ is now the mean epicyclic radius, and $I_{l}(x)$ is the Bessel function of imaginary argument. The dispersion relation (9) reduces to the standard Lin-Shu one when the nonaxisymmetric contribution proportional to $\tan \psi$ vanishes. Comparison with the standard Lin-Shu dispersion relation for tightly wrapped waves (Toomre 1964; Lin \& Shu 1966; Lin et al. 1969; Shu 1970; Binney \& Tremaine 1987, p. 360) shows that our replacement of $k_{r}$ by $k$ and $k_{*}$ gives a dispersion relation which is reasonably correct for much open waves. See Morozov (1980, 1981a,b), Griv \& Peter (1996), Griv (1998), and Griv et al. (1999) for a discussion.

The dispersion relation (9) is complicated. A further simplification results from restricting the frequency range of the waves examined by taking the low-frequency limit, $\left|\omega_{*}\right|<\kappa$ (Griv et al. 2000). As a result, the terms in series (8) and (9) for which $|l| \geq 2$ may be neglected, and consideration will be limited to the transparency region between the inner $\left(\omega_{*}+\kappa=0\right)$ and outer $\left(\omega_{*}-\kappa=0\right)$ Lindblad resonances. Secondly, we pay attention mainly to the long-wavelength oscillations, $x \approx$ $k_{*}^{2} \rho^{2} \leq 1$, the case of epicyclic radius small compared with wavelength. (But, of course, in order to be appropriate for a WKB wave we consider the perturbations with $\left|k_{r}\right| R \gg 1$.) In this limit, one can use the following asymptotic expansion of the modified Bessel functions $I_{0}(x) \approx 1+x^{2} / 4, I_{1}(x) \approx x / 2$, and $\exp (-x) \approx 1-x+x^{2} / 2$. See Griv et al. (2000) for a discussion. As a result, from dispersion relation (9) one determines the frequency of the most important low-frequency $\left(\left|\omega_{*}\right| \lessgtr \kappa\right)$ and long-wavelength $\left(k_{*}^{2} \rho^{2} \lesssim 1\right)$ Jeans oscillations:

$\omega_{* 1,2} \approx \pm p\left|\omega_{\mathrm{J}}\right|-i v_{\mathrm{c}}$,

where

$\omega_{\mathrm{J}}^{2} \approx \kappa^{2}-2 \pi G \sigma_{0}|k|\left\{1+\left[(2 \Omega / \kappa)^{2}-1\right] \sin ^{2} \psi\right\} F(x)$

is the squared Jeans frequency, $x \leq 1, F(x) \approx \mathrm{e}^{-x}$ is the socalled "reduction factor," $F(x) \rightarrow 1$ in a dynamically cold system $\left(c_{\mathrm{r}} \rightarrow 0\right)$ and decreases with increasing $x$ (increasing the velocity spread) in a dynamically hot disk (Griv et al. 2000). In Eq. (10), $p=1$ for Jeans-stable $\left(\omega_{\mathrm{J}}^{2}>0\right)$ disturbances and $p=i$ for Jeans-unstable $\left(\omega_{\mathrm{J}}^{2}<0\right)$ ones, the term involving $v_{\mathrm{c}}$ is the small correction, and in general $\left|\omega_{\mathrm{J}}\right| \sim \Omega$. Accordingly, in the gravitationally stable system $\left(\omega_{\mathrm{J}}^{2}>0\right)$ the Jeans oscillations are the natural ones $\left(\mathfrak{R} \omega_{* 1,2} \neq 0\right.$ and $\left.\mathfrak{J} \omega_{* 1,2}=0\right)$. In the gravitationally unstable disk $\left(\omega_{\mathrm{J}}^{2}<0\right)$ they are gravity-unstable disturbances which grow aperiodically, that is, in the unstable range $\mathfrak{J} \omega_{* 1,2}>0$ and $\mathfrak{R} \omega_{* 1,2}=0$. This is the classical gravitational Jeans-type instability. As is seen from Eq. (10), rare interparticle collisions lead to the weak damping of both Jeansunstable and Jeans-stable modes of oscillations. This is physically obvious: as a result of interparticle collisions (and thermal pressure), the organized motion tends to be lost. Evidently, in the considered case of rare collisions $\left(v_{\mathrm{c}}<\kappa\right)$ viscous forces resulting from interparticle collisions are not sufficient to damp gravity-unstable disturbances.

In Eq. (11),

$\Lambda=\left\{1+\left[(2 \Omega / \kappa)^{2}-1\right] \sin ^{2} \psi\right\} \approx\left(1+3 \sin ^{2} \psi\right)$.

This factor indicates an extra $(\Lambda>1)$ clumping associated with the azimuthal forces $(\psi \neq 0)$ in the differentially rotating $(2 \Omega / \kappa>1)$ media. Lau \& Bertin (1978) and Lin \& Lau (1979) have obtained a somewhat similar expression for the extra clumping in a gas dynamical model. From Eq. (10), the growth rate of the instability is high,

$\mathfrak{J} \omega_{*} \approx \sqrt{2 \pi G \sigma_{0}|k| \Lambda F(x)-\kappa^{2}}$,

$\mathfrak{J} \omega_{*} \lesssim \kappa$, and in general $\mathfrak{J} \omega_{*} \sim \Omega$. That is, the instability develops rapidly on a rotation time scale. The expression (12) indicates the tendency of growth of the particle clumping with increasing $\psi$ (this is because $\Lambda \propto \psi$ ).

Thus, nonaxisymmetric (spiral) Jeans-type disturbances in a nonuniformly rotating system are more difficult to suppress than the axisymmetric (radial) ones, in general agreement with the work by Goldreich \& Lynden-Bell (1965), Julian \& Toomre (1966), and Toomre (1981). The result is quite obvious: spiral disturbances, in contrast with radial ones, are subject to the influence of the differential character of the rotational motion. The free kinetic energy associated with the differential rotation of the system under study is one possible source for the growth of the energy of these spiral Jeans-type disturbances, and appears to be released when angular momentum is transferred outward. It is interesting to note that Maxwell (1859) considered just this kind of spiral instabilities with $m=1$ in his study concerning the stability of the Saturnian uniform rings whose radial extent was considerably larger than the average interparticle distance. Maxwell made a correct that, in such a system, the azimuthal force resulting from azimuthal displacements was more important in determining the stability than was the radial force resulting from radial displacements. See Lau \& Bertin (1978, p. 513) for a discussion.

The Jeans disturbances can be stabilized by the random velocity spread. Morozov (1980, 1981a,b), Griv \& Peter (1996), Griv (1998), Griv et al. (1999, 2000) have obtained the modified stability criterion to suppress the instability of all but not 
only axisymmetric Jeans-type disturbances:

$c_{\mathrm{r}} \geq c_{\text {crit }} \approx c_{\mathrm{T}}\left\{1+\left[(2 \Omega / \kappa)^{2}-1\right] \sin ^{2} \psi\right\}^{1 / 2} \approx 2 c_{\mathrm{T}}$,

or Toomre's $Q \equiv c_{\mathrm{r}} / c_{\mathrm{T}}$ parameter

$Q \geq Q_{\text {crit }} \approx 2 \Omega / \kappa \approx 2$,

respectively. Here, $c_{\mathrm{T}}=3.4 G \sigma_{0} / \kappa$ is the well-known Safronov-Toomre critical velocity dispersion (Safronov 1960, 1980; Toomre 1964) to suppress the instability of axisymmetric disturbances. ${ }^{3}$ Similar stability criterion can also be derived from the Lynden-Bell \& Kalnajs (1972, Eq. (A11) therein) dispersion relation for open spirals.

It would be natural to suppose that as a disk of mutually gravitating particles evolves it should arrive at a state near the limit of stability, so that the mean particle radial-velocity dispersion would come close to the modified critical value $c_{\text {crit }}$ as given by Eq. (13). Interestingly, analysis of the Voyager 2 data (Lane et al. 1982) for the B ring of Saturn shows that $Q \approx 2$, i.e., the $\mathrm{B}$ ring is located near the boundary of Jeans stability. This could be indicative of a self-gravitation mechanism for generating fine structure in Saturn's rings. The structure is created if self-gravity is included; only collisions do not create the structure. The spiral arms in nonuniformly rotating systems are a mechanism for angular momentum transfer (Lynden-Bell \& Kalnajs 1972). Thus, the Safronov-Toomre critical velocity dispersion $c_{\mathrm{T}}$ should stabilize only axisymmetric (radial) disturbances of the Jeans type. The differentially rotating disk is still unstable against nonaxisymmetric (spiral) Jeans-type disturbances (Lau \& Bertin 1978, Figs. 6 and 7 therein). Hence, for a nonuniformly rotating thin system of mutually gravitating particles the spiral gravity disturbances are more important in determining the stability than are the radial disturbances studied by Safronov (1960, 1980), Toomre (1964), Lin \& Shu (1966), Lin et al. (1969), Shu (1970), and Genkin \& Safronov (1975).

The fact that self-gravitating nonuniformly rotating particulate disks can exhibit strong nonaxisymmetric responses even when the axisymmetric stability criterion is fulfilled, was most convincingly demonstrated already by Julian \& Toomre (1966), concerning the gravitational effect of any single orbiting mass concentration (such as gas "lumps" in galaxies). Similar result for self-gravitating gas disk was obtained by Goldreich \& Lynden-Bell (1965). Julian \& Toomre's calculations showed that even a stable stellar system (possessing a velocity dispersion more than sufficient for local radial gravitational stability) to be remarkably responsive in a spiral-like manner to localized forcing. To emphasize it again, these forced spiral waves are not to be confused, of course, with Lin \& Shu's fully self-consistent density wave proposal explored in our theory. In Toomre (1981) this amplification was discussed in terms of "swing-mechanism," very reminiscent of the way we reach the nonaxisymmetric stability criterion (13). The role of swingamplification in the generation of quasi-steady spiral structure

3 To obtain Eq. (13), one first finds the critical wavenumber $k_{\text {crit }}$ from the relation $\partial \omega_{\mathrm{J}}^{2} / \partial k=0$. Then this $k_{\text {crit }}$ is substituted into the dispersion relation and from the condition $\omega_{\mathrm{J}}^{2} \geq 0$ the critical velocity dispersion $c_{\text {crit }}$ is found. in galaxies was discussed in Sellwood \& Carlberg (1984), emphasizing also the crucial importance of cooling in opposing the heating due to spirals themselves.

The growth rate of the instability has a maximum at the wavelength

$\lambda_{\text {crit }}=2 \pi / k_{\text {crit }} \approx(4 \pi \Omega / \kappa) \rho \approx 2 \pi h$

(Griv et al. 2000). It means that of all harmonics of initial disturbance, one disturbance with $\lambda_{\text {crit }} \approx 2 \pi h$ and $\mathfrak{J} \omega_{*} \sim \Omega$ will be formed asymptotically in time of $2-3$ rotations. For the parameters of Saturn's rings, $\lambda_{\text {crit }} \leq 100 \mathrm{~m}$.

An important issue not addressed in the paper is the spatial inhomogeneity of the disk. We have shown above that for the homogeneous disk Jeans-unstable disturbances grow aperiodically, thus perhaps leading one to believe that we treat Jeans disturbances as being localized near corotation $\left(\mathfrak{R} \omega_{*}=\right.$ 0 ). However, for the realistic inhomogeneous disk, it may be shown that Jeans-unstable disturbances grow in an oscillatory fashion with the small real part of $\omega_{*}$ proportional to the inverse radial scale of spatial inhomogeneity $\left|(\partial / \partial r) \ln \left(2 \Omega \sigma_{0} / \kappa c_{\mathrm{r}}^{2}\right)\right|^{-1}$ and the azimuthal mode number $m$ (Griv et al. 2000, 2002).

\section{Quasilinear equations}

Evidently, the unstable fluidlike Jeans oscillations must influence the distribution function of the main, nonresonant part of particles in such a way as to hinder the wave excitation, i.e., to increase the random velocity spread ${ }^{4}$. This is because the Jeans instability, being essentially a gravitational one, tends to be stabilized by random motions of particles. Therefore, along with the growth of the oscillation amplitude the velocity dispersion must increase, and finally in the disk there can be established a stationary distribution so that the Jeans-unstable disturbances are completely vanishing.

To repeat ourselves, the nonlinear nonresonant waveparticle interaction increases the velocity dispersion of particles. Subsequently, sufficient velocity dispersion given by Eq. (13) prevents the Jeans instability from occuring. By reducing the magnitude of the relative velocity of particles, the dissipative collisions cause a new Jeans instability elsewhere in the disk. The latter may lead to a recurrent instability cycle. There seems no reason why this instability cycle should not occur in Saturn's rings. Interestingly, in the Saturnian ring system on a small scale the irregular rings have been observed to be undergoing variation and oscillations with time and ring longitude (Smith et al. 1982). Esposito (1986, 1993) especially noted that most irregular microstructures are likely much younger than

\footnotetext{
${ }^{4}$ The Jeans instability associated with departures of macroscopic quantities from the thermodynamic equilibrium is algebraic in nature and has nothing to do with any resonant effects; a relatively simple hydrodynamical model can also be used to investigate the instability (Lau \& Bertin 1978: Lin \& Lau 1979). Such hydrodynamic instabilities distinguish themselves in that a rather large group of so-called nonresonant particles takes part in their generation; the Jeans instabilities are generated by almost all the particles of the phase space. Morozov (1978) has discussed the problem of quasilinear saturation of Jeans instability in a pure gaseous disk.
} 
the solar system and new rings are created by some unknown mechanisms.

We substitute the solution (8) into Eq. (4) and average the latter over time. As a result we obtain the equation for the slow part of the distribution function:

$$
\frac{\partial f_{0}}{\partial t}=i \frac{\pi}{2} \sum_{k} \mathcal{E}_{k} \frac{\partial}{\partial v_{\perp}} \frac{k_{*} \kappa}{\chi v_{\perp}} \sum_{l=-\infty}^{\infty}\left[\frac{l^{2} J_{l}^{2}(\chi)}{\omega_{*}-l \kappa}-\frac{l^{2} J_{l}^{2}(\chi)}{\omega_{*}^{*}-l \kappa}\right] \frac{\partial f_{0}}{\partial v_{\perp}}
$$

where $\mathcal{E}_{\boldsymbol{k}}=\left|\Phi_{\boldsymbol{k}}\right|^{2} \exp \left(2 \mathfrak{J} \omega_{*} t\right)$ and $\omega_{*}^{*}$ is the complex conjugate frequency of excited waves.

As usual in the quasilinear theory, in order to close the system one must engage an equation for $\mathcal{E}_{\boldsymbol{k}}$. Averaging over the fast oscillations, we obtain

$(\partial / \partial t) \mathcal{E}_{k}=2 \mathfrak{J} \omega_{*} \mathcal{E}_{k}$.

Equations (15) and (16) form the closed system of weakly nonlinear equations for Jeans oscillations of the rapidly rotating homogeneous disk of particles, and describe a diffusion in velocity space. The spectrum of oscillations $\Re \omega_{*}$ and their growth/damping rate $\mathfrak{J} \omega_{*}$ are determined by Eqs. (9) and (10) of the linear theory.

Two general physical conclusions can be deduced without solving Eq. (15). First, the initial distribution of particles will change upon time under the action of only growing disturbances $\left(\mathfrak{J} \omega_{*}>0\right)^{5}$. Second, the distribution of random velocities will change under the action of both the radial $\propto k_{r}$ and the azimuthal $\propto k_{\varphi} \equiv m / r$ forces (this is because in Eq. (15) $\left.k_{*} \propto k=\sqrt{k_{r}^{2}+k_{\varphi}^{2}}\right)$.

In the following, we restrict ourselves to the most important long-wavelength and low-frequency disturbances, $\chi^{2} \ll 1$, $x^{2} \ll 1$, and $|l| \leq 1$ (Griv et al. 1999; Griv et al. 2000; Sect. 3 above). Then in Eq. (15) one can use the expansion $J_{1}^{2}(\chi) \approx \chi^{2} / 4$. Equation (15) takes the simple form

$\partial f_{0} / \partial t=\mathcal{D}_{v}\left(\partial^{2} f_{0} / \partial v_{\perp}^{2}\right)$

where $\mathcal{D}_{v}=\left(\pi / 2 \kappa^{2}\right) \sum_{k} k_{*}^{2} \mathfrak{J} \omega_{*} \mathcal{E}_{k}$, both $\mathcal{E}_{k}$ and $\mathfrak{J} \omega_{*}$ are functions of $t$. As is seen, the velocity diffusion coefficient for nonresonant particles $\mathcal{D}_{v}(t)$ is independent of $\boldsymbol{v}_{\perp}$ (to lowest order). This is a qualitative result of the off-resonant, or adiabatic (Sagdeev \& Galeev 1969, p. 67) character of the particles' interaction with almost aperiodically growing collective aggregates.

An expression for $f_{0}$ at $t \rightarrow \infty$ can be deduced from Eq. (17). We introduce the standard substitution $\mathrm{d} \tau / \mathrm{d} t=\mathcal{D}_{v}(t)$ and $\mathrm{d} / \mathrm{d} t=(\mathrm{d} \tau / \mathrm{d} t)(\mathrm{d} / \mathrm{d} \tau)$. Equation (17) can be written as

$\partial f_{0} / \partial \tau-\partial^{2} f_{0} / \partial v_{\perp}^{2}=0, \quad \partial \mathcal{D}_{v} / \partial \tau=2 \mathfrak{J} \omega_{*}$.

The partial solution of the system (18) is

$f_{0}\left(v_{\perp}, \tau\right)=\frac{\text { const. }}{\sqrt{\tau+c_{r}^{2} / 2}} \exp \left[-\frac{v_{\perp}^{2}}{4\left(\tau+c_{r}^{2} / 2\right)}\right]$.

${ }^{5}$ In interaction with gravitationally stable waves, $\mathfrak{J} \omega_{*}<0$ or $\mathfrak{J} \omega_{*} \rightarrow 0$, with the exception of spatially small resonant regions, there is no energy exchange in the wave-particle system (Lynden-Bell \& Kalnajs 1972; Griv 1996; Griv \& Peter 1996).
Accordingly, the energy of the oscillation field $\propto \sum_{k} k_{*}^{2} \mathcal{E}_{k}$ plays the role of a "temperature" in the nonresonant-particle distribution. As the disturbance energy increases, the initial distribution spreads $\left(f_{0}\left(v_{\perp}^{2}\right)\right.$ becomes less peaked), and the effective temperature $T$ grows with time (i.e., Gaussian spread increases): $T \propto \int \mathcal{D}_{v}(t) \mathrm{d} t \propto \sum_{k} k_{*}^{2} \mathcal{E}_{k}=2 \tau$. The diffusion in velocity space is due entirely to the growth of the Jeans-unstable disturbances in a self-gravitating system subject to a time-dependent potential. The Jeans-unstable modes increase the velocity dispersion of the main, nonresonant part of the particle distribution, ultimately at the expense of circular motion and/or gravitational energy. The diffusion, however, presumably tapers off as Jeans stability is approached: the radial velocity dispersion $c_{\mathrm{r}}$ becomes greater than the critical one given by Eq. (13). The relaxation time scale is short $\sim\left(\mathfrak{J} \omega_{*}\right)^{-1} \gtrsim \Omega^{-1}$, i.e., comparable with $2-3$ periods of the disk rotation. Formally, the relaxation corresponds to the presence of a collision term in the right-hand member of Eq. (17) to describe the "encounters" between the particles and the growing gravity disturbances.

Thus the gravitational Jeans-type instability of a disk will in fact be suppressed because of the efficient "heating" of the medium during the growth of that instability. This statement becomes more plausible if we recognize that the density $\delta U$ of the perturbed potential energy is a negative quantity. Clearly as gravitational instability develops the amplitude $|\delta U|$ of the unstable harmonics will grow, setting energy free to heat the medium. This process - the self-suppression of instability by a rise in the amplitude of the unstable harmonics - recalls the case of nonresonant quasilinear relaxation in plasmas, which can effectively heat the medium without raising the entropy (Sagdeev \& Galeev 1969, p. 67; Galeev \& Sagdeev 1983). The diffusion in velocity space is due entirely to the growth of the Jeans-unstable disturbances in a self-gravitating system subject to a time-dependent potential. Eventually, after 2-3 rotations the disk evolves toward a quasi-stationary, marginally Jeansstable distribution. Such a situation naturally implies a finite lifetime for the fine spiral structure, and a source of cooling is required to maintain spirality. The velocity diffusion of nonresonant particles takes place because they gain wave energy as the instability develops. By this relaxation, the system is led to a quasiequilibrium. The system, however, does not evolve significantly once the system reaches the quasi-steady state with $c_{\mathrm{r}} \approx c_{\text {crit }} \approx 2 c_{\mathrm{T}}$ (or $Q \approx 2$, respectively).

\section{Conclusions}

Summarizing the results of Sects. 3 and 4, we may say that the gravitational Jeans-type instability of small-amplitude gravity disturbances may be considered as an effective generating mechanism for fine density wave structure in Saturn's rings. However, the off-resonant nonlinear interaction between the Jeans-unstable gravity disturbances and particles effectively increases the spread of random velocities of the bulk of Saturn's rings particles. This collisionless relaxation mimics the usual thermal relaxation, leading to Maxwell-Boltzmann type velocity distributions with an effective temperature (random velocity dispersion) that increases with time. The diffusion presumably tapers off as Jeans stability is approached: $c_{\mathrm{r}}$ becomes greater 
than $c_{\text {crit }} \approx 2 c_{\mathrm{T}}$ (or Toomre's parameter $Q \gtrsim 2$, respectively), where $c_{\mathrm{T}}$ is the ordinary Safronov-Toomre critical velocity dispersion to suppress the instability of only axisymmetric gravity disturbances. Computer $N$-body experiments have shown similar behavior for a hot system of particles repeatedly (Salo 1992, 1995; Richardson 1994; Osterbart \& Willerding 1995; Sterzik et al. 1995; Griv 1998; Daisaka \& Ida 1999; Ohtsuki \& Emori 2000). The relaxation time scale is short $\sim \Omega^{-1}$. Thus, the spiral wake structure is time dependent and transient, being created and destroyed on the time scale of an order of Keplerian period. Rare $\left(v_{\mathrm{c}}<\Omega\right)$ inelastic (dissipative) collisions between particles are necessary for the reconstruction of the wavelike $\sim 100 \mathrm{~m}$ structure ${ }^{6}$. This fine density wave structure of the order of $2 \pi$ times the typical thickness in Saturn's rings may be a long-term recurrent phenomenon. The structure is governed primarily by gravity. The result is in agreement with suggestions of Griv et al. (2000).

At the present we do not have observations with resolution comparable to the scale height of the main rings (Showalter \& Nicholson 1990; Horne \& Cuzzi 1996). The stability analysis presented here would have to be regarded as a prediction of the long-term ring structure based on theoretical modeling that could be compared to future Cassini spacecraft measurements - recurrent spiral density waves with size and spacing between them in the range of few tens to few hundreds meters ${ }^{7}$.

Lastly, we add a brief comment on our work. In local computer simulations by Salo, Richardson, Osterbart \& Willerding, Sterzik et al., Griv, Daisaka \& Ida, and Ohtsuki \& Emori, one can see spiral wave fragments (cylindric structures of $100 \mathrm{~m}$ or so) with a definite pitch angle with respect to the local shear flow. This angle is almost about $\psi_{\text {crit }}=20^{\circ}-25^{\circ}$. Even though the analysis presented here shows that there is a dominant nonaxisymmetric $(\psi \neq 0)$ Fourier mode of maximum instability with $\lambda_{\text {crit }} \approx 100 \mathrm{~m}$ in Saturn's rings, at the present we cannot explain the angle $\psi_{\text {crit }}$ in the local WKB version of our theory. In the local WKB approximation it is assumed that the wave vector and the wavefrequency vary continuously. We will show in the next paper of the series by utilizing the more accurate nonlocal WKB approximation that in fact the characteristic oscillation frequencies of an inhomogeneous disk must be "quantized," i.e., must pass through a discrete series of values. According to the WKB method the spectrum of frequencies $\omega$ is determined by the quasiclassical rules of Bohr-Sommerfeld quantization:

$\int_{r_{1}}^{r_{2}} k_{r}\left(r^{\prime}\right) \mathrm{d} r^{\prime}=\left(n+\frac{1}{2}\right) \pi$

${ }^{6}$ Schmit \& Tscharnuter (1995, 1999), Schmidt et al. (2001), and Salo et al. (2001) suggested a viscous instability as a generating mechanism for structure formation in the densest central portions of Saturn's B ring with $\tau>1$.

7 The Cassini Ultra-Violet Imaging Spectrograph, or UVIS observations include stellar occultations. Numerous diffraction-limited star occultations by the rings are a prime objective for the UVIS (Esposito et al. 1998). The $2 \mathrm{~ms}$ integration period in this mode will give a ring radial resolution of better than $20 \mathrm{~m}$. The latter will make possible to observe waves, wakes, and ring edges-all characteristics of ring dynamics and history. where $r_{1}, r_{2}$ are the "reflection" points (say, the inner and outer Lindblad resonances) and $n=0,1,2, \cdots$ (see, e.g., Alexandrov et al. 1983, p. 249). Equation (20) has a simple physical meaning: there must be place for an integer number of halfwaves between the turning points in the transparency range of the medium between $r_{1}$ and $r_{2}$. Equation (20) implies that $\omega$ is independent of $r$; that is, the spiral-wave front is not distorted by differential rotation. The pattern is composed of one or more modes, and discrete modes are stationary in a rotating frame and hence do not wind up $^{8}$. It seems likely that such an approach will allow us to determine the critical angle $\psi_{\text {crit }}$. Further, the nonlocal theory provides a formal basis for the main idea that formed the basis for the local description, namely, that of short-wavelength $\left(\left|k_{r}\right| L \gg 1\right.$, where $L$ is the radial scale of spatial inhomogeneity; Griv et al. 2000, 2002) approximation. The work is in progress.

Acknowledgements. We thank Tzi-Hong Chiueh, David Eichler, Edward Liverts, Frank Shu, Irina Shuster, Raphael Steinitz for valuable discussions, and the first referee of the paper, Eugen Willerding, for constructive criticism that improved our understanding of the problems under consideration. This work was supported in part by the Israel Science Foundation, the Israeli Ministry of Immigrant Absorption, and the Academia Sinica in Taiwan. One of us (E.G.) appreciates the hospitality of the ASIAA, where the work was begun.

\section{Appendix A: Conditions for the applicability of the quasilinear theory}

A very important feature of the instability under consideration is the fact that it is aperiodic (the real part of the Dopplershifted wavefrequency $\mathfrak{R} \omega_{*}$ vanishes in a circular rotating reference frame). Usually (e.g., Galeev \& Sagdeev 1983; Lifshitz \& Pitaevskii 1981; Swanson 1989) the quasilinear theory is used when the condition that the growth rate be small compared with the real part of wavefrequency $\left(\left|\mathfrak{J} \omega_{*} / \mathfrak{R} \omega_{*}\right| \ll 1\right)$ is satisfied. However, as shown first by Shapiro \& Shevchenko (1964), the quasilinear theory can be applied also to aperiodic instabilities, provided that in the initial state the plasma parameters deviate little from the critical values at which instability sets in. See Shapiro \& Shevchenko (1964) and Sagdeev \& Galeev (1969, p. 67) for a discussion.

Let us obtain conditions for the applicability of quasilinear theory of Jeans instability in Saturn's main rings with rare interparticle collisions. The rapidly developing distribution function $f_{1}(\boldsymbol{r}, \boldsymbol{v}, t)$ in Eq. (3) depends on the "background" slowly developing distribution function $f_{0}(\boldsymbol{v}, t)$. To find the specific form of this dependence we use two assumptions that are customary in the quasilinear theory. First of all, we assume that one can neglect the nonlinear interaction between different harmonics of the collective motions (Sagdeev \& Galeev 1969; Galeev \& Sagdeev 1983). The latter condition is that the correction $f_{1}$

\footnotetext{
8 The weakly inhomogeneous approximation used throughout this paper has the meaning that the discrete spectrum will differ little from a continuous spectrum, and in the zeroth-order approximation of the theory may be regarded as continuous.
} 
(Eq. (8)) to the distribution function be small in comparison with the function $f_{0}$ :

$\left|\frac{\partial f_{0}}{\partial\left(v_{\perp}^{2} / 2\right)} \sum_{l=-\infty}^{\infty} \Phi_{1} \frac{l \kappa J_{l}^{2}(\chi)}{l \kappa-\omega_{*}-i v_{\mathrm{c}}}\right| \ll f_{0}$,

where $\omega_{*} \equiv i \mathfrak{J} \omega_{*}, \mathfrak{J} \omega_{*}>0$, and $v_{\mathrm{c}} \ll \mathfrak{J} \omega_{*}$. For the most important low-frequency oscillations with $\mathfrak{J} \omega_{*} \lesssim \kappa$ (that is, $|l| \leq 1$ ) and long-wavelength oscillations with $\chi \lesssim 1$ (that is, $k_{*} \lesssim \kappa / c_{\mathrm{r}}$ ), it is thus necessary that

$\epsilon\left|\Phi_{1}\right| / c_{\mathrm{r}}^{2} \ll 1$,

where $\epsilon \approx k_{*}^{2} c_{\mathrm{r}}^{2} / \kappa^{2} \lesssim 1$. The condition (A.2) has a simple physical significance: the density of wave-field gravitational energy must be less than (or comparable to) the density of thermal kinetic energy of particles (cf. Lifshitz \& Pitaevskii 1981).

Secondly, it is also assumed that the change in the distribution function with time, brought about by the reaction of the oscillations, takes place slowly, so that

$\left|\frac{1}{f_{0}} \frac{\partial f_{0}}{\partial t}\right| \ll\left|\frac{1}{f_{1}} \frac{\partial f_{1}}{\partial t}\right|$.

In other words, we assume that the variation of $f_{0}$ is adiabatic. In Eq. (A.3), making use of Eq. (8), one obtains

$\left|\frac{1}{\left(\omega_{*}+i v_{\mathrm{c}}-l \kappa\right) f_{0}} \frac{\partial f_{0}}{\partial t}\right| \ll 1$

where $l= \pm 1, \pm 2, \cdots$. In the case under consideration, when the instability is aperiodic $\left(\mathfrak{R} \omega_{*}=0, \mathfrak{J} \omega_{*}>0\right.$, and $\left.\mathfrak{J} \omega_{*} \lesssim \kappa\right)$, we obtain the strongest condition when $l= \pm 1$. Inasmuch as $\left|f_{0}^{-1} \partial f_{0} / \partial t\right| \lesssim \mathfrak{J} \omega_{*}$ (Eq. 17), this condition can be written in the form

$$
\frac{\mathfrak{J}^{2} \omega_{*}}{\mathfrak{J}^{2} \omega_{*}+\kappa^{2}} \ll 1 .
$$

From Eq. (A.5) it follows that this condition is satisfied when $\mathfrak{J} \omega_{*}<\kappa$. The growth rate $\mathfrak{J} \omega_{*}$ of Jeans instability will be small $\left(\mathfrak{J} \omega_{*}<\kappa\right.$, where $\kappa \approx \Omega$ ) if (Eq. (12))

$$
\frac{\left|\kappa^{2}-8 \pi G \sigma_{0}\right| k|F(x)|}{\kappa^{2}} \ll 1 \text { and } 8 \pi G \sigma_{0}|k| F(x)>\kappa^{2} \text {, }
$$

i.e., when the deviation from the critical disk parameters at which the instability sets in is small, $\left|\omega_{*}\right|<\kappa$ and $k_{*} \rho \sim 1$. In this event oscillations will be excited with wavelengths $\lambda$ in a narrow range around the value $\lambda_{\text {crit }} \approx 2 \pi h$ (Eq. (14)). We regard many harmonics with randomly distributed phases as being excited in this range of wavelengths.

\section{References}

Alexandrov, A. F., Bogdankevich, L. S., \& Rukhadze, A. A. 1984, Principles of Plasma Electrodynamics (Berlin: Springer)

Araki, S. 1991, Am. Sci., 79, 44

Bertin, G., \& Lin, C. C. 1996, Spiral Structure in Galaxies: A Density Wave Theory (Cambridge: MIT)

Binney, J., \& Tremaine, S. 1987, Galactic Dynamics (Princeton: Princeton Univ. Press)

Bridges, F. G., Hatzes, A. P., \& Lin, D. N. C. 1984, Nature, 309, 333

Colombo, G., Goldreich, P., \& Harris, A. 1976, Nature, 264, 344
Cook, A. F., \& Franklin, F. A. 1964, AJ, 69, 173

Cuzzi, J. N., Lissauer, J. J., \& Shu, F. H. 1981, Nature, 292, 703

Daisaka, H., \& Ida, S. 1999, Earth Planets Space, 51, 1195

Dones, L., Cuzzi, J. N., \& Showalter, M. R. 1993, Icarus, 105, 184

Drummond, W. E., \& Pines, D. 1962, Nuclear Fus. Suppl., 3, 1049

Durisen, R. H. 1995, Icarus, 115, 65

Esposito, L. W. 1986, Icarus, 67, 345

Esposito, L. W. 1993, AREPS, 21, 487

Esposito, L. W., Colwell, J. E., \& McClintock, W. E. 1998, P\&SS, 46, 1221

Franklin, F. A., \& Colombo, G. 1978, Icarus, 33, 279

Franklin, F. A., Cook II, A. F. R. T. F., Barrey, et al. 1987, Icarus, 69, 280

Galeev, A. A., \& Sagdeev, R. Z. 1983, in Handbook of Plasma Physics, ed. M. N. Rosenbluth, \& R. Z. Sagdeev (Amsterdam: NorthHolland), 1, 679

Genkin, I. L., \& Safronov, V. S. 1975, SvA, 19, 189

Goertz, C. K., \& Morfill, G. 1988, Icarus, 74, 325

Goldreich, P., \& Lynden-Bell, D. 1965, MNRAS, 130, 125

Goldreich, P., \& Tremaine, S. 1978, Icarus, 34, 227

Goldreich, P., \& Tremaine, S. 1982, ARA\&A, 20, 249

Griv, E. 1996, P\&SS, 44, 579

Griv, E. 1998, P\&SS, 46, 615

Griv, E., \& Chiueh, T. 1997, A\&A, 311, 1033

Griv, E., \& Chiueh, T. 1998, ApJ, 503, 186

Griv, E., Gedalin, M., \& Eichler, D. 2001, ApJ, 555, L29

Griv, E., Gedalin, M., Eichler, D., \& Yuan, C. 2000, P\&SS, 48, 679

Griv, E., Gedalin, M., \& Yuan, C. 1997, A\&A, 328, 531

Griv, E., Gedalin, M., \& Yuan, C. 2002, A\&A, 383, 338

Griv, E., \& Peter, W. 1996, ApJ, 469, 84

Griv, E., Rosenstein, B., Gedalin, M., \& Eichler, D. 1999, A\&A, 347, 821

Grivnev, E. M. 1988, SvA, 32, 139

Hohl, F. 1978, AJ, 83, 768

Horne, L. J., \& Cuzzi, J. N. 1996, Icarus, 119, 285

Jeffreys, H. 1947, MNRAS, 107, 263

Julian, W. H., \& Toomre, A. 1966, ApJ, 146, 810

Krall, N. A., \& Trivelpiece, A. W. 1986, Principles of Plasma Physics (San Francisco: San Francisco Press)

Lane, A. L., Hord, C. W., R. A. West, et al. 1982, Science, 215, 537

Lau, Y. Y., \& Bertin, G. 1978, ApJ, 226, 508

Lewis, M. C., \& Stewart, G. R. 2000, AJ, 120, 3295

Lifshitz, E. M., \& Pitaevskii, L. P. 1981, Physical Kinetics (Oxford: Pergamon)

Lin, C. C., \& Lau, Y. Y. 1979, SIAM J. App. Math., 60, 97

Lin, C. C., \& Shu, F. H. 1966, Proc. Natl. Acad. Sci., 55, 229

Lin, C. C., Yuan, C., \& Shu, F. H. 1969, ApJ, 155, 721

Lissauer, J. J., Shu, F. H., \& Cuzzi, J. N. 1981, Nature, 292, 707

Lynden-Bell, D., \& Kalnajs, A. J. 1972, MNRAS, 157, 1

Maxwell, J. C. 1859, in Sci. Papers, 1960, vol. 1, ed. W. D. Niven, 288 (New York: Dover)

Morozov, A. G. 1978, SvA Lett., 4, 115

Morozov, A. G. 1980, SvA, 24, 391

Morozov, A. G. 1981a, SvA Lett., 7, 109

Morozov, A. G. 1981b, SvA, 25, 421

Nicholson, P. D., French, R. G., Tollestrup, E., et al. 2000, Icarus, 145, 474

Ohtsuki, K., \& Emori, H. 2000, AJ, 119, 403

Osterbart, R., \& Willerding, E. 1995, P\&SS, 43, 289

Poulet, F., Sicardy, B., Dumas, C., Jorda, L., \& Tiphene, D. 2000, Icarus, 145,147

Richardson, D. C. 1994, MNRAS, 269, 493

Sagdeev, R. Z., \& Galeev, A. A. 1969, Nonlinear Plasma Theory, ed. T. M. O’Neil, \& D. L. Book (New York: Benjamin) 
Safronov, V. S. 1960, Ann. d'Astrophys., 23, 979

Safronov, V. S. 1980, in Early Solar System Processes and the Present Solar System, ed. D. Lal (Amsterdam: North-Holland), 73

Salo, H. 1992, Nature, 359, 619

Salo, H. 1995, Icarus, 117, 287

Salo, H., Schmidt, J., \& Spahn, F. 2001, Icarus, 153, 295

Schmidt, J., Salo, H., Spahn, F., \& Petzschmann, O. 2001, Icarus, 153, 316

Schmit, U., \& Tscharnuter, W. M. 1995, Icarus, 115, 304

Schmit, U., \& Tscharnuter, W. M. 1999, Icarus, 138, 173

Sellwood, J. A., \& Carlberg, R. G. 1984, ApJ, 282, 61

Shapiro, V. D., \& Shevchenko, V. I. 1964, Sov. Phys.-JETP, 18, 1109

Showalter, M. R., \& Nicholson, P. D. 1990, Icarus, 87, 285

Shu, F. H. 1970, ApJ, 160, 99

Shu, F. H. 1984, in Planetary Rings, ed. R. Greenberg, \& A. Brahic, (Tucson: Univ. of Arizona Press), 513

Shu, F. H., Cuzzi, J. N., \& Lissauer, J. J. 1983, Icarus, 53, 185
Shu, F. H., \& Stewart, G. R. 1985, Icarus, 62, 360

Smith, B. A., Soderblom, R. R., Batson, et al. 1982, Science, 215, 504 Spahn, F., \& Sremcevic, M. 2000, A\&A, 358, 368

Sterzik, M. F., Herold, H., Ruder, H., \& Willerding, E. 1995, P\&SS, 43,259

Stewart, G., Lin, D. N. C., \& Bodenheimer, P. 1984, in Planetary Rings, ed. R. Greenberg, \& A. Brahic (Tucson: Univ. of Arizona Press), 447

Swanson, D. G. 1989, Plasma Waves (Boston: Academic Press)

Toomre, A. 1964, ApJ, 139, 1217

Toomre, A. 1981, in Structure and Evolution of Normal Galaxies, ed. S. M. Fall, \& D. Lynden-Bell, 111 (Cambridge: Cambridge Univ. Press)

Vandervoort, P. O. 1970, ApJ, 161, 87

Vedenov, A. A., Velikhov, E. P., \& Sagdeev, R. Z. 1962, Nuclear Fus. Suppl., 2, 465 